\title{
Microplastics in surface waters of the Wei River, China
}

\author{
Jie Jiao ${ }^{1, \mathrm{a}}$ Hong $\mathrm{Hu}^{\mathrm{a}, *}$ Gang Chen ${ }^{\mathrm{b}, *}$, and Zechuan Yang ${ }^{\mathrm{a}}$ \\ ${ }^{a}$ College of Environmental Science and Engineering, Ocean University of China, Qingdao, 266100, China \\ ${ }^{\mathrm{b}}$ College of Marine Life science, Ocean University of China, Qingdao, 266100, China \\ *Corresponding author. Tel/Fax: +86 532 82031809. chengang@ouc.edu.cn (G. Chen), College of Marine Life science, Ocean University \\ of China, No. 5, Yu Shan Road, Qingdao, Shandong Province 266003, China; Tel/Fax: +86 532 66786381. E-mail address: \\ hhu@ouc.edu.cn (H. Hu), College of Environmental Science and Engineering, Ocean University of China, No.238, Song Ling Road, \\ Qingdao, Shandong Province 266100, China
}

\begin{abstract}
Microplastics are a new type of persistent organic pollutants, usually on the micron scale. In this study, we investigated the abundance, distribution, and other characterization of microplastics in surface waters of the Wei River Basin in the Shandong peninsula. The results showed that the abundance of microplastics in the surface water of Wei River varied from 0.40 to 1.20 items/L, and the average abundance was 0.81 items $/ \mathrm{L}$, which was at a moderate pollution level compared with other rivers. It was found that the abundance of microplastics was higher in densely populated areas, and hydrodynamic conditions such as river inflow and seawater scour also affected the abundance of microplastics. Fiber (83.4\%) was the dominant type. Colorless $(93.6 \%)$ was the dominant color type. The particle size $(0.06-0.5 \mathrm{~mm})(47.9 \%)$ was the main size of microplastics in the Wei River. This study provides data for the further study of microplastics in rivers and provides a warning for the management and prevention of microplastics in freshwater.
\end{abstract}

\section{Introduction}

Thomas et al. first proposed the concept of microplastics in 2004 in journal Science ${ }^{[1]}$. Microplastics are small plastic particles, usually on the micron scale. Microplastics have the large specific surface areas and are easy to enrich other pollutants such as PCBs and PBDEs ${ }^{[2]}$. Many studies have found microplastics in fish and shellfish $^{[3,4]}$. Microplastics, which are difficult to degrade and bioaccumulative, can be passed through the food chain and can ultimately be hazardous to human health ${ }^{[5]}$.

The wide distribution of microplastics has always been the focus of research. Microplastics are now found in oceans ${ }^{[6]}$, lakes ${ }^{[7]}$, rivers ${ }^{[3,8]}$ and even drinking water ${ }^{[9]}$. For river microplastics, researchers initially studied the estuary area. With the deepening of the research, the research on microplastics in inland rivers has also been gradually carried out, such as Wei River ${ }^{[8]}$ and Hai River ${ }^{[10]}$. However, the research data on microplastics in river surface water is still scarce.

In this study, 17 stations along the mainstream of Wei River were selected and surface water samples from each station were collected. Through the experimental process of filter digestion and microscopic observation, the abundance, particle size, shape, and color of microplastics at each station were counted. Through data analysis, the distribution and pollution characteristics of microplastics in the Wei River Basin were obtained, which provided a basis for the study of the transport and migration behaviors of microplastics through rivers to the ocean.

\section{Materials and methods}

\subsection{Study area}

Wei River is a river from the Shandong Peninsula flowing into Laizhou Bay. It is approximately $233 \mathrm{~km}$ long and has a basin area of $64,493.2 \mathrm{~km}^{2}$. Wei River has two sources. The north source originates from Guanzhuang Township of Yishui County, and the south source originates from the Longwangmiao Township of $\mathrm{Ju}$ County. After the confluence of the two sources, it turns north and flows through Zhucheng, Gaomi, Anqiu, Fangzi, Changyi, and other counties and districts to the Laizhou Bay. There are many tributaries in the Wei River. The two large reservoirs through which Wei River flows are Qiangkuang Reservoir and Xiashan Reservoir.

The sampling time of this study was from October 1 to 4,2020 . Sampling sites were selected at two sources ( $\mathrm{S} 01$, $\mathrm{S} 02$ ), the confluence of the two sources ( $\mathrm{S} 03)$, the confluence of important tributaries (S08, S09, S10, S13, $\mathrm{S} 16)$, the areas flowing through cities and villages ( $\mathrm{S} 05$, S06, S07, S14, S15), the reservoirs that are flowing through (S04, S11, S12), and the estuary (S17) of Wei River, a total of 17 stations (Fig. 1.).

*Corresponding author: chengang@ouc.edu.cn (G. Chen), hhu@ouc.edu.cn (H. Hu) 


\subsection{Sample collection}

Three parallel samples were selected from each station at a distance of $100 \mathrm{~m} .50 \mathrm{~L}$ of river surface waters were collected with a glass water sampler and filtered through a plankton net ( $64 \mu \mathrm{m}$ mesh size). The outer wall of the net was washed repeatedly so that the microplastics would not be attached to the inner wall of the net. The water samples left in the bottom collector were collected in glass bottles and kept away from light.

\subsection{Sample preparation}

The sampling preparation method of the water sample is by the previous method and some improvements have been made ${ }^{[8]}$. Firstly, the water sample in the sampling bottle is filtered by a diaphragm vacuum pump with a 0.45 $\mu \mathrm{m}$ filter membrane; Then, the filter membrane was rinsed repeatedly with $30 \% \mathrm{H}_{2} \mathrm{O}_{2}$, and the rinsing solution was collected into a conical flask. Finally, $0.1 \mathrm{~g} \mathrm{FeCl}_{2}$ was added and placed in a water bath at $60^{\circ} \mathrm{C}$ for digestion for $24 \mathrm{~h}$. The purpose of this step is to remove organic matter and other substances that may affect the subsequent microscopic observation.

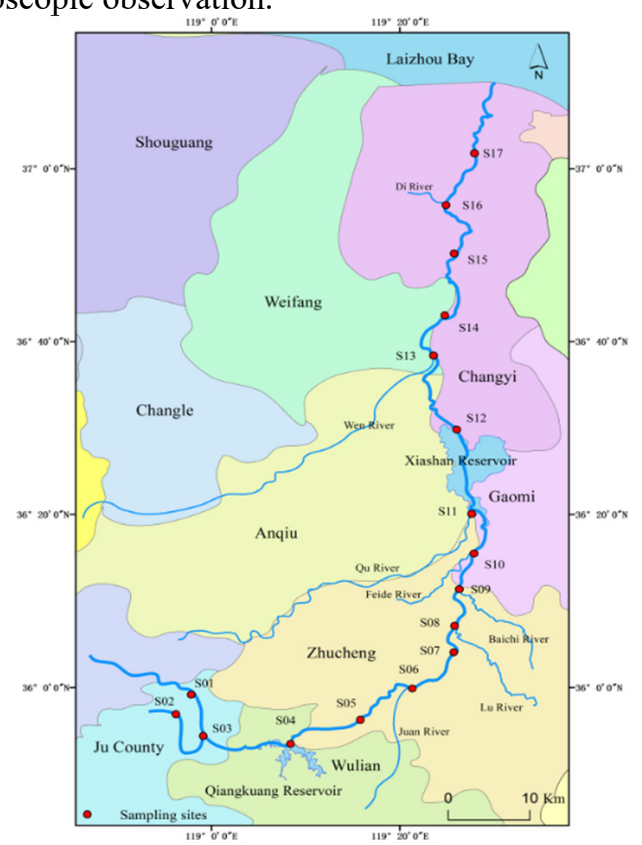

Fig. 1. Sampling sites in Wei River

\subsection{Observation and identifification of microplastics}

The digested solution in the conical flask was filtered through with a series of sieves apertures: $2 \mathrm{~mm}, 1 \mathrm{~mm}$, $0.5 \mathrm{~mm}, 0.1 \mathrm{~mm}$, and $0.01 \mathrm{~mm}$ in turn. The samples from the screen were carefully and thoroughly rinsed with ultrapure water into petri dishes, and each dish represented each microplastic size range. After drying the petri dishes, the number, color, shape, and particle size of microplastics in each dish were observed and counted with a microscope.

\subsection{Statistical analysis}

The unit of microplastics abundance in Wei River surface waters was items/L (number of microplastics in the surface water per liter). Data and descriptions were analyzed using Microsoft Excel 2010 and SPSS vs. 22 (IBM).

\section{Results and discussion}

\subsection{Abundance of microplastics}

The abundance of microplastics in the surface water of Wei River ranged from 0.4 to 1.2 items $/ \mathrm{L}$, and the average abundance was 0.81 items/L (Fig. 2.), which was lower than that in Wei River (3.67-10.7 items/L) ${ }^{[8]}$, Pearl River $(0.379-7.924 \text { items } / \mathrm{L})^{[11]}$, and West River $(2.99-9.87$ items $/ \mathrm{L})^{[12]}$, and higher than in the Yulin River $\left(1.3 \times 10^{-}\right.$ ${ }^{2}$ items $\left./ \mathrm{L}\right)^{[13]}$, Tuojiang River $(0.912 \pm 0.2-3.40 \pm 0.707$ items $/ \mathrm{L})^{[14]}$, and Han River $\left(0-4.29 \times 10^{-2} \text { items } / \mathrm{L}\right)^{[15]}$. On the whole, Wei River microplastics pollution level in the medium level of pollution.

The microplastics abundance of each sampling station in Wei River has obvious regional differences. S01 and S02 are the northern and southern sources of the Wei River respectively. The surrounding ecological environment of the $\mathrm{S} 01$ sampling station is better than that of $\mathrm{S} 02$, and the water mobility is stronger, so the microplastics abundance is lower than that of S02. Some scholars believe that hydrodynamic conditions have a great influence on the abundance of microplastics ${ }^{[16]}$. S03 is the confluence of the two sources, so the abundance of microplastics is higher than S01 and S02. S04 is located at the confluence of Wei River and other rivers into the Qiangkuang reservoir, so the abundance of microplastics has increased again. Many scholars believe that the confluence of rivers is an important source of microplastics in the mainstream ${ }^{[15}$, 17]. The abundance of microplastics decreased at S05, which is speculated to be because the site is located at the exit of the Qiangkuang reservoir, and the microplastics are diffused and diluted through the reservoir. S06, S08, S13, and S15 are where tributaries and main streams meet, so the abundance of microplastics is higher than that of the previous site. S08 is the site with the highest microplastics abundance among all the sites. The reasons are: (1) tributary inflow; (2) electroplating factory nearby may produce microplastics waste into the river; (3) sampling site is located under the bridge, which may be affected by traffic. The microplastics abundance of S13 is also high, mainly because the Wei River flows through Anqiu City, and city Non-point source pollution on the microplastics in the river has been recognized by some scholars ${ }^{[18]}$. Significant variance analysis was conducted for the microplastic abundance of sampling stations (S06, S07, S15) near cities and stations near rural areas (S01, S05, S16). The results showed that the abundance of microplastics in urban areas was significantly higher than that in rural areas $(p<0.05)$, indicating that the higher the level of economic development, the higher the density of population, the more serious the microplastics pollution ${ }^{[18]}$. The abundance of microplastics in the estuary area (S17) 
decreased obviously, which may be due to the dilution of microplastics by seawater exchange. Previous research data on the abundance of microplastics in Laizhou Bay $\left(1.7 \times 10^{-3} \pm 1.5 \times 10^{-3} \text { items } / \mathrm{L}\right)^{[6]}$ are lower than the data in this study, which can prove this conjecture.

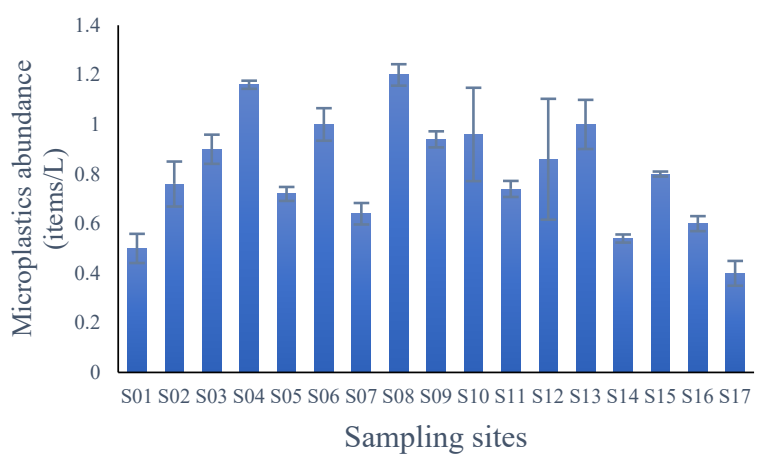

Fig. 2. Abundance distribution of microplastics collected from the Wei River.

\section{2 characteristic of microplastics}

The microplastics were divided into three types as fiber (83.4\%), foam (2.2\%), fragment (14.4\%) (Fig. 3 a). The conclusion is consistent with the majority of research results, that is, fibrous microplastics account for the majority of the literature ${ }^{[6,8]}$. Fibrous microplastics are mainly from clothing washing. Each wash produces more than 1,900 fibrous microplastics ${ }^{[19]}$. Wei River flows through many cities and villages, and the villagers' washing wastewater may be an important source of fibrous microplastics. Besides, the use of ropes, fishing nets, carpets, and other processes can also produce fibrous microplastics.

The colors of microplastics in the Wei River includes colorless $(93.6 \%)$, red $(2.8 \%)$, black $(2.2 \%)$, and blue $(1.6 \%)$ (Fig. 3. b). One of the reasons for the largest proportion of colorless microplastics is that there is more agricultural land around the Wei River basin. The colorless plastic film used in the planting industry and the plastic cloth used in vegetable greenhouses are the main sources of colorless microplastics. The other reason is that microplastics could fade after photodegradation, aging, and other physical and chemical effects ${ }^{[16]}$. Another possible reason is that river life, such as fish, prefers the colored microplastic to eat ${ }^{[20]}$.

In this study, microplastics are classified into 5 categories: $0.06-0.1 \mathrm{~mm}(14.1 \%), 0.1-0.5 \mathrm{~mm}(47.9 \%)$, $0.5-1 \mathrm{~mm}(17.5 \%), 1-2 \mathrm{~mm} \quad(12.5 \%)$ and $>2 \mathrm{~mm}(7.9 \%)$ (Fig. 3. c). Except for $0.06-0.1 \mathrm{~mm}$, the smaller the particle size, the larger the proportion. Through comparison, it is found that $0.1-0.5 \mathrm{~mm}$ microplastics also occupy a large proportion in other rivers, such as Wei River $(<0.5 \mathrm{~mm}, 68.1 \%)^{[8]}$, Hai River $(0.1-1 \mathrm{~mm}$, $54.1 \%)^{[10]}$, Yulin River $(0.064 \sim 0.25 \mathrm{~mm},>80 \%)^{[13]}$, Fengshan River $(0.05-0.297 \mathrm{~mm},>60 \%)^{[3]}$, and Han River $(0.1-1 \mathrm{~mm},>90 \%)^{[15]}$, which reflects the common characteristics of microplastics in rivers: microplastics with a particle size of $0.1-0.5 \mathrm{~mm}$ have the largest content in rivers.

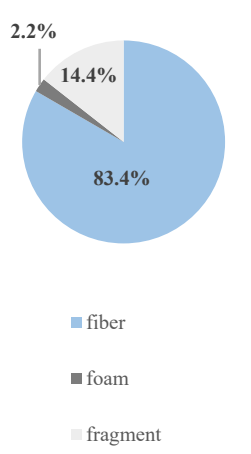

(a)

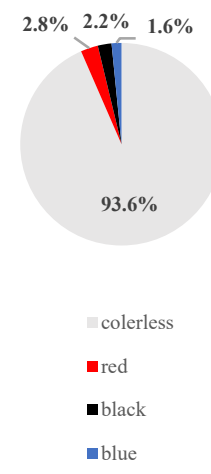

(b)

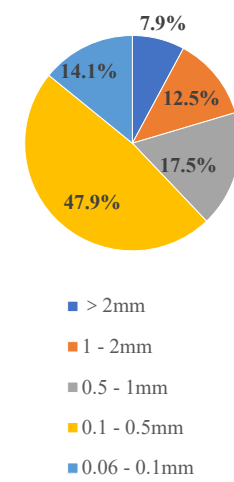

(c)
Fig. 3. characteristic of microplastics collected from the Wei River. (a) shape (b) color (c) particle size

\section{Conclusion and Prospect}

Microplastics are widely found in the surface waters of the Wei River, ranged from 0.4 to 1.2 items/L, and the average abundance was 0.81 items $/ \mathrm{L}$, which is at a moderate pollution level compared with other rivers. The inflow of tributaries, the density of population and industry, and the hydrodynamic affect all affect the abundance of microplastics in the Wei River.

Colorless (93.6\%) and fiber (83.4\%) microplastics account for the vast majority in Wei River, which indicates that residents' activities are an important source of microplastics. By comparison, it was found that the most common size of microplastics in rivers was $0.1-0.5 \mathrm{~mm}$.

Although the survey scope and the number of experimental samples in this study are limited, it is sufficient to indicate that microplastics exist in large quantities in rivers and other freshwater systems, which should be paid enough attention to. Future research should also focus on the identification of pollution sources and the migration and transformation of microplastics.

\section{Acknowledgments}

The study was financially supported by the National Major Special Project for Water Pollution Treatment and Control "Technology Integration of Watershed Ecological Function Zoning Management" Project (2017ZX07301001).

\section{References}

1. Thompson R. C. Lost at sea: where is all the plastic? J. Science, 304, 838 (2004)

2. Yeo B. G., Takada H., Yamashita R., et al. PCBs and PBDEs in microplastic particles and zooplankton in open water in the Pacific Ocean and around the coast of Japan. J. Mar. Pollut. Bull.,151, 110806 (2020)

3. Tien C., Wang Z., Chen C. S. Microplastics in water, sediment and fish from the Fengshan River system: Relationship to aquatic factors and accumulation of polycyclic aromatic hydrocarbons by fish. J. Environ. Pollut., 265, 114962 (2020) 
4. Ding J., Li J., Sun C., et al. An examination of the occurrence and potential risks of microplastics across various shellfish. J. Sci. Total Environ., 739, 139887 (2020)

5. Qu H., Ma R., Barrett H., et al. How microplastics affect chiral illicit drug methamphetamine in aquatic food chain? From green alga (Chlorella pyrenoidosa) to freshwater snail (Cipangopaludian cathayensis). J. Environ. Int., 136,105480 (2020)

6. Teng J., Zhao J., Zhang C., et al. A systems analysis of microplastic pollution in Laizhou Bay, China. J. Sci. Total Environ., 745,140815 (2020)

7. Wang W., Yuan W., Chen Y., et al. Microplastics in surface waters of Dongting Lake and Hong Lake, China. J. Sci. Total Environ., 633, 539-545 (2018)

8. Ding L., Mao R. F., Guo X., et al. Microplastics in surface waters and sediments of the Wei River, in the northwest of China. J. Sci. Total Environ., 667, 427434 (2019)

9. Makhdoumi P., Amin A. A., Karimi H., et al. Occurrence of microplastic particles in the most popular Iranian bottled mineral water brands and an assessment of human exposure. J. J. Water Process Eng., 39,101708 (2021)

10. Liu Y., Zhang J., Cai C., et al. Occurrence and characteristics of microplastics in the Haihe River: An investigation of a seagoing river flowing through a megacity in northern China. J. Environ. Pollut., 262,114261 (2020)

11. Lin L., Zuo L., Peng J., et al. Occurrence and distribution of microplastics in an urban river: A case study in the Pearl River along Guangzhou City, China. J. Sci. Total Environ., 644, 375-381 (2018)

12. Huang D., Li X., Ouyang Z., et al. The occurrence and abundance of microplastics in surface water and sediment of the West River downstream, in the south of China. J. Sci. Total Environ., 756, 143857 (2021)

13. Mao Y., Li H., Gu W., et al. Distribution and characteristics of microplastics in the Yulin River, China: Role of environmental and spatial factors. J. Environ. Pollut., 265, 115033 (2020)

14. Zhou G., Wang Q., Zhang J., et al. Distribution and characteristics of microplastics in urban waters of seven cities in the Tuojiang River basin, China. J. Environ. Res., 189, 109893 (2020)

15. Park T., Li S. L. Occurrence of microplastics in the Han River and riverine fish in South Korea. J. Sci. Total Environ., 708, 134535 (2020)

16. Qi, H., Wang, Y., Zhang, D., et al. Microplastics in Moshui River Sediment: Abundance, Morphology and Spatail Distribution. J. Transactions and Oceanology and Limnology, 3, 69-77 (2019)

17. Fraser M. A., Chen L., Ashar M., et al. Occurrence and distribution of microplastics and polychlorinated biphenyls in sediments from the Qiantang River and Hangzhou Bay, China. J. Ecotox. Environ. Safe., 196, $110536(2020)$

18. Xiong X. Wu C. Elser J. J. et al. Occurrence and fate of microplastic debris in middle and lower reaches of the Yangtze River - From inland to the sea. J. Sci. Total Environ., 659, 66-73 (2019)

19. Browne M. A., Crump P., Niven S. J., et al. Accumulation of microplastic on shorelines woldwide: sources and sinks. J. Environ. Sci. Technol., 45, 9175-9179 (2011)

20. Zhao S, Zhu L, Li D. Microplastic in three urban estuaries, China. J. Environ. Pollut., 206, 597-604 (2015) 